\section{Combating bacteria}

Ecasol, an alternative to the use of costly and aggressive chemicals to combat the risk of bacteria, viruses, protozoa or fungi, is available for the first time in the UK from Cannon Ecaflo, a division of hygiene company Cannon.

Ecaflo devices harness electro-chemical activation technology (ECA) to produce a highly effective Ecasol solution which combats potentially deadly microbes. These are a constant threat in both private and public healthcare facilities, in particular dental surgeries where the risk of contaminated water lines and the build up of biofilm is high due to the micro-bore water supply systems used.

The Ecaflo dental system is effective against all known spores and microorganisms including Legionella and MRSA. It works by passing a precisely formulated salt-water solution through a specially developed, flow-through electrolytic reactor which creates an intense electrical field. This results in the instant transformation of the solution into a powerful, biocidal solution.

Reader response number 51

\section{High quality scans}

The Iluma Cone-Beam CT Scanner from Kodak provides dental professionals with the CT technology needed to produce high resolution CT scans in the practice at a considerable cost and time saving to the patient.

The scanner provides full-cranial, highly accurate 3D radiographic images for the more accurate diagnosis and treatment planning for dental procedures including orthodontics, implantology, airway assessment and oral and orthognathic surgery.

Undistorted, anatomically correct views of jaws, teeth and facial bones along with cross-sectional, axial, coronal, sagittal, cephalometric and panoramic views are easily generated with the Iluma cone-beam technology.

Reader response number 52

\title{
Interdental cleaning with convenience
}

Flix is a new disposable interdental stick which uses patented technology to adhere to each stick a fine woven mesh of floss. This will then deliver fluoride as it comes into contact with saliva.

With its tapered design and ergonomic handle, Flix is the ideal solution for cleaning interdentally, especially around orthodontic braces and implants. It is available in patient packs of 100 sticks and chairside packs of 500 (ideal for patients to sample). It is available in 'Traditional', 'Mint Sensation' and 'Zero' - (fluoride free) options.

Reader response number 50

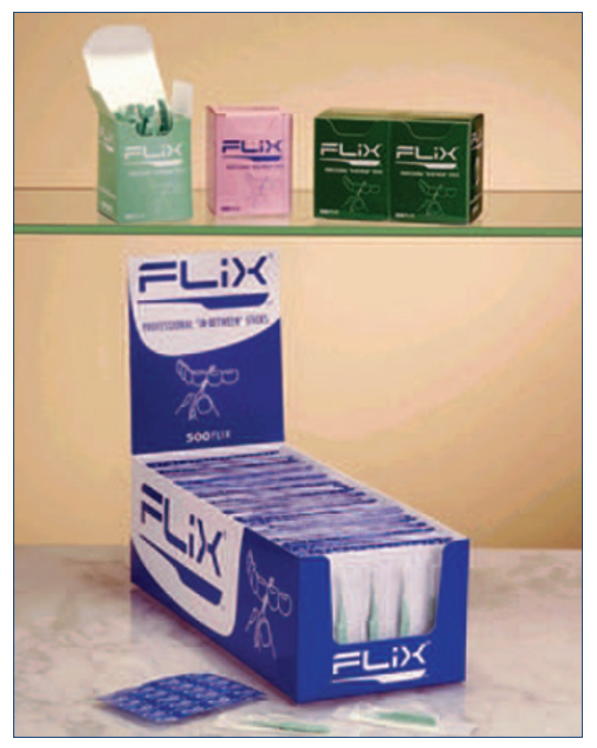

\section{Disposing of clinical waste}

Econix Ltd, a new company based in Sheffield, has just launched its newest product range called Bio-bin. Constructed from paper, Bio-bin is a new generation of non-sharp, clinical waste containers aimed at replacing plastic containers currently used in the industry.

Bio-bin has a 96\% paper construction which is completely water tight and leak proof and has passed United Nations 3291 safety testing. The bins have been designed to stack inside each other, which makes them very

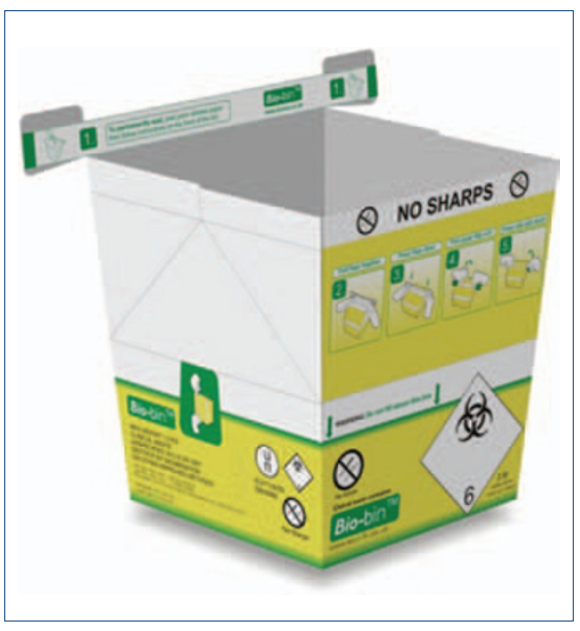

space efficient to store. Because of their square base they are also very stable and store waste more efficiently then round containers .

They come with their own built in lid and have easy to follow user instructions printed on each bin. The weight of the bins is also less then that of the equivalent plastic bins. This reduces the weight of waste that is produced which in turn will also reduce disposal and incineration costs.

Bio-bin is available in sizes ranging from 21 up to 301 and can be sent straight to incineration with out autoclaving. (However, some waste produce companies have policies in place that insist on autoclaving all waste before it leaves the premises.)

Another unique feature of the Bio-bin is that once sealed it can be placed in an autoclave. As the temperature and steam increase it attacks the adhesive that seals the bin and breaks it down. This opens the bin inside the autoclave automatically and allows the contents to be treated. At the end of the process the bins are still intact and can be removed easily for final disposal.

Reader response number 53 


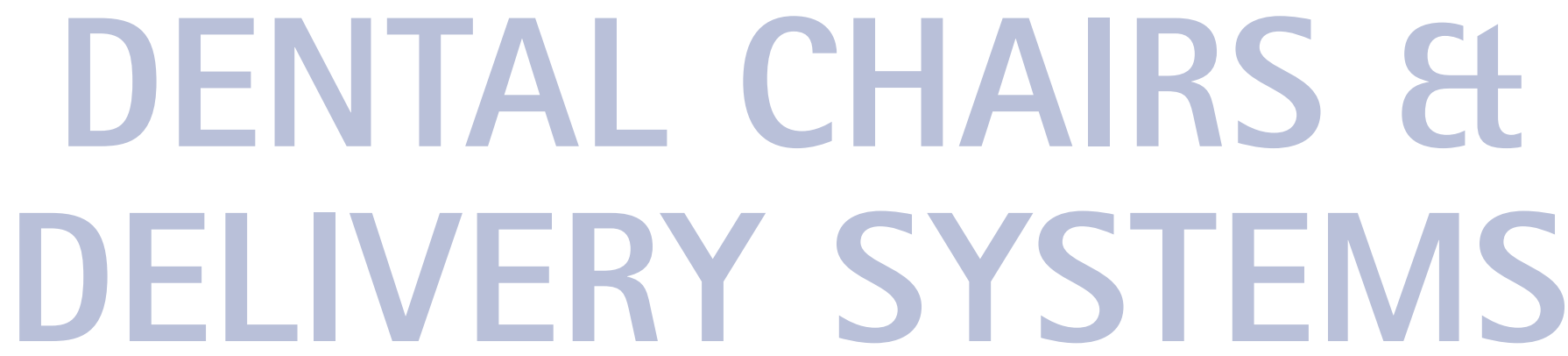

\section{Ergonomical design}

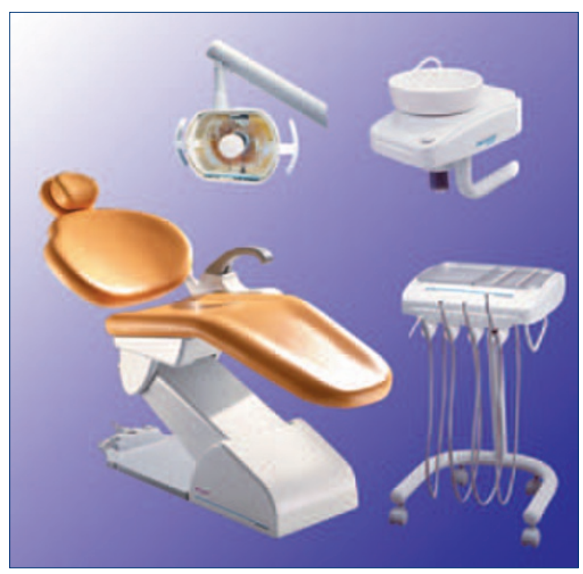

Ergonomically designed for maximum comfort, the Galla chair features a large backrest with sculptured anatomical curvature for excellent patient support. Three work positions can be pre-programmed and zero-position return, reflector, backrest and seat movements are all controlled by the foot-switch. Its smooth lines help effective cleaning and decontamination.

A built-in safety device immediately interrupts the lowering movement to prevent accidents and the stabilising bar in the base and the armoured elevation lever ensure the stability of the chair.

The Flex Cart $\mathrm{S}$ is lightweight and mobile. It has three handpiece terminals for Midwest or Borden handpieces and a three-in-one syringe attachment for water, air and mist. The cart has its own asepsy flush and clean bottled water systems for complete biosafety, as well as a pneumatic activation block for integration with electronic components.

A package of Dabi Atlante surgery equipment, consisting of a Galla chair, cuspidor, light and Flex Cart, including professional fitting and installation by Tower Dental Equipment, is available from the company.

Reader response number 55

\section{Customised treatment centres}

Takara Belmont's state-of-the-art dental equipment range includes the Clesta II, Cleo, Voyager and Phot-X.

Treatment centres are customised to suit the clients' needs and can include choices such as left or right handed dentistry to single or four-handed use with specific flexi-arm opportunities. Focus on surgery décor is ever increasing and a wide range of attractive pastel and black coloured upholstery is on offer as well as coordinated cuspidor bowls to complement chair upholstery and clinic décor.

The Clesta II is available with the new soft touch upholstery and ultra-deep padding. The low-tilt chair comes with a close up synchronised axis backrest that matches extension and tilt to reduce patient angle and maximise comfort and support. The SP-Cleo dental chair has a unique Belmont folding leg, offers easy access for patients of any age from the front or either side of the chair. This allows treatment staff the room to work freely and permits face-to-face consultation for a more personal approach.

The Voyager II L dental system offers a totally flexible yet elegant and prac-

\section{Flexible fit chair}

Manufactured in Finland and renowned for their durability Fimet F1 dental chairs are available in knee-break and flat bed versions in soft vinyl upholstery with a choice of 18 colours.

The F1 Soft Knee-break is a very flexible chair that can fit into the tightest surgery without compromising on comfort. Economical and oil free, the chairs are driven by two quiet DC motors with one gear for power lift and one for the back rest with a $150 \mathrm{~kg}$ lifting capacity. The chairs feature a joystick foot control with eight $(2 \times 4)$ positioning programs,

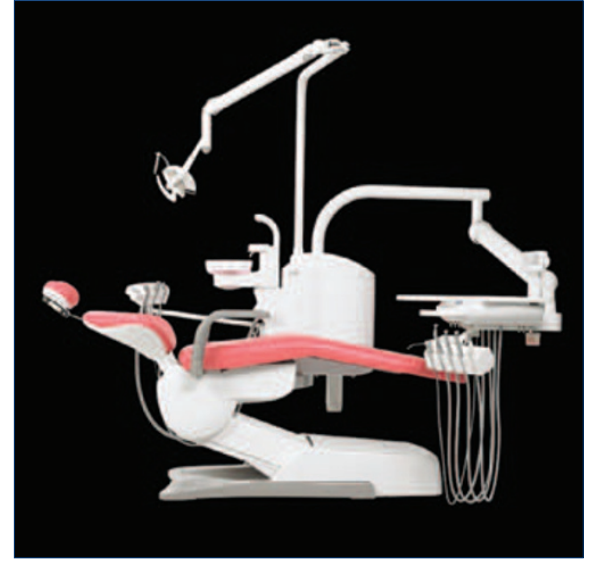

tical dental system. It enables right or left-handed dentistry with the use of a pivoting doctor's table and cuspidor which can be easily positioned on either side of the dental chair. The height of the doctor's table can also be adjusted. The Phot-X is a multi function DC X-ray machine using high frequency technology that reduces ray dosage to the skin with optimum radiation levels for every diagnostic need.

Reader response number 54

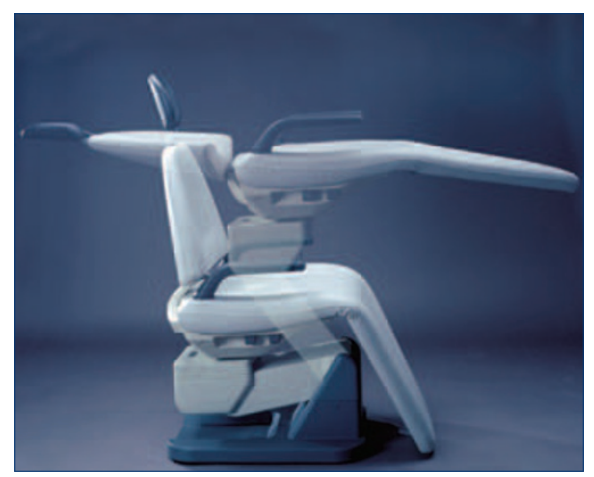

double articulating horseshoe headrests and clean armrests.

Reader response number 56 
Upright posture

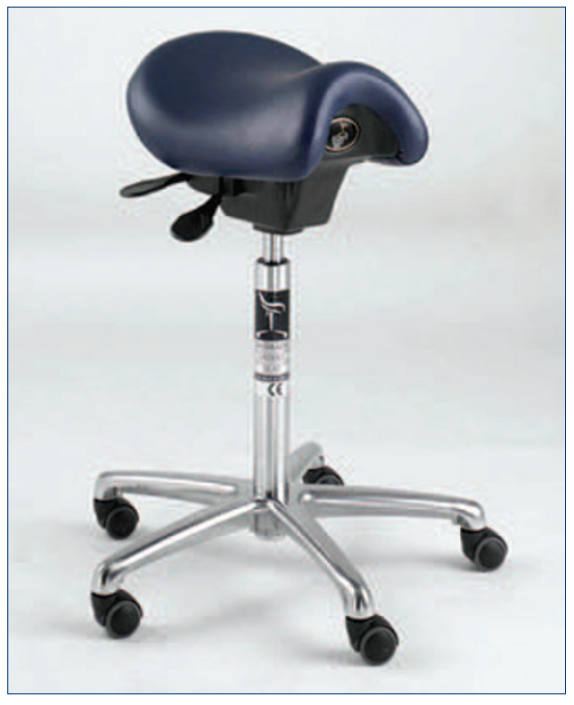

The unique Bambach saddle stool has a special design which provides an anterior pelvic tilted position to keep the spine straight, encouraging the natural lumbar curve and discouraging sitting in the forward slump, on one buttock or fidgeting from side to side. This upright posture will help prevent musculoskeletal disorders in the future whilst addressing current concerns, according to the company. The Bambach seat's swivel base houses the central height adjustment gas piston which, when locked, allows a small amount of 'bounce' for added comfort. The base is fitted with easyglide castors which allow you to 'scoot' around the surgery with effortless ease and a wide variety of finishes and colours are designed to suit all tastes.

Reader response number 57

\section{Wipe-clean surfaces}

The Osmond Group seating solutions include the Jumper and Amazone saddle seats, the Barrage saddle seat with additional back-rest, the Luna and Orbit fully adjustable, comfort stools and the Vario stool with 'front rest' or back rest.

All models are designed and constructed to the same high quality and have a comprehensive variety of adjustments to suit all sizes and preferences. In addition, the options of free moving or brakeable castors, a range of colours complementary to surgery equipment and décor and hygienic, wipe-clean surfaces make these stools and seats ideal for today's dentists and professional support staff.

Reader response number 58

\section{Ease of integration}

Sirona's range of treatment centres includes the $\mathrm{C}+$ range, designed to maximise flexibility, integration and technology within the dental practice, creating a seamless workflow that optimises productivity.

Integration into the surrounding dental environment is simple and sophisticated.

By networking your entire practice through the treatment centre's integrated patient communication system, it then becomes the hub for patient education, displaying digital radiography, scheduling and even entertaining.

All controlled through a single footswitch, the control of a three way syringe, air or electric driven handpieces, intraoral camera, prophylaxis,

\section{Full back support}

Henry Schein's surgery equipment range includes the Pelton and Crane Ellipse dental units.

The Ellipse accommodates left or right handed dentists with equal facility, the chair also swivels on its axis allowing for specialist treatment, and all chair

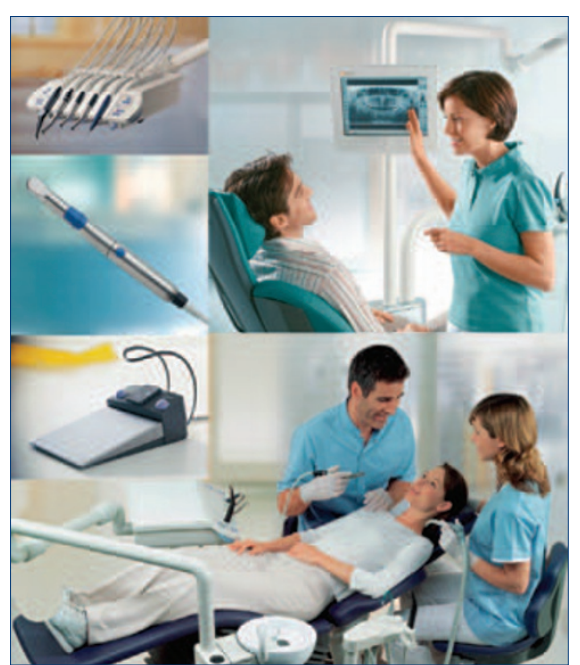

an ultrasonic scaler and the LED curing light is at your fingertips.

Reader response number 59

controls are easily accessible by the operator or the nurse. Features include the ErgoSoothe fully integrated lumbar and back massaging system, soft and ultra-durable Ultraleather upholstery, and the operating light features the ideal feathered-edge pattern to reduce eye fatigue.

Reader response number 60 